\title{
Cutaneous adverse reactions of COVID-19 vaccines: a systematic review
}

\author{
Ahmadreza Shamsabadi \\ Mohammad Hossein Golezar \\ Abbas Mardani \\ Manthar Ali Mallah \\ Samira Golezar \\ Bagher Moradi \\ Kowsar Qaderi ( $\nabla$ kowsar.qaderi@gmail.com ) \\ Kermanshah University of Medical Sciences
}

\section{Systematic Review}

Keywords: vaccines, COVID-19, SARS-CoV-2, adverse event, exanthema, dermatologic, skin, cutaneous, Coronavirus, Cutaneous Adverse Events, Manifestations

Posted Date: October 25th, 2021

DOl: https://doi.org/10.21203/rs.3.rs-1000625/v1

License: (1) This work is licensed under a Creative Commons Attribution 4.0 International License. Read Full License 


\section{Abstract}

Background: Numerous vaccines are implementation for the prevention of severe course and lethal outcome of coronavirus disease 2019 (COVID-19).

Objective: This systematic review aims to summarize and integrated the findings of studies regarding cutaneous side effects of COVID-19 vaccines.

Methods: This systematic review conducted by searching the scientific databases of PubMed, Scopus, Science direct, and Web of knowledge from the beginning of the COVID-19 to10/5/2021. Articles were reviewed and analyzed based on the Preferred Reporting Items for Systematic Reviews and MetaAnalyses (PRISMA) checklist.

Results: Seventeen studies were included after the screening of search results based on to the eligibility criteria. The results showed that injection site reactions and delayed large local reactions were the most common side effects arising from all vaccine types. Most cutaneous reactions occurred in middleaged women, after the first dose of vaccine, and 1-21 days after vaccination. Most cutaneous reactions were self-limiting, need little or no therapeutic intervention, and were not regarded as a barrier to injecting a second dose.

\section{Limitations:}

Conclusion: In certain groups such as patients with allergies and a history of local injection reactions, pre-vaccination counseling and the use of appropriate medications may be helpful. However, more studies are needed to investigate the side effect profile of all COVID-19 vaccines.

\section{Introduction}

The pandemic of coronavirus disease 2019 (COVID-19) has prompted the fast development and licensing of vaccinations against the pathogen responsiblesevere acute respiratory syndrome coronavirus 2 (SARS-CoV-2) (1). Vaccination candidates were created by more than 100 businesses and universities throughout the world, utilizing both well-established and more experimental vaccine platforms (2). More than a year later, a range of efficacious and safe COVID-19 vaccinations are now being administered across the world. Currently licensed vaccines use nucleic acid-based vaccination platforms, such as messenger ribonucleic acid (mRNA), viral vector platforms (using various adenovirus strains), and inactivated virus. Protein subunit vaccines have not yet been included into mass immunization campaigns, although they may do so soon. Given the scarcity of vaccines and the pressing necessity for mass immunization utilizing billions of doses in the face of the ongoing epidemic, there is substantial regional variation in the usage of the various agents. Many nations lack access to COVID-19 vaccines, despite the fact that certain high-income countries have already immunized the vast majority of their citizens. Because trade names may differ locally, we will always refer to vaccinations by their generic names.

Antiviral immunity is induced effectively by humoral and cellular immune responses. Apart from those that employ live attenuated virus, most vaccination types require many doses and/or adjuvants to effectively stimulate the innate immune system, which then elicits adaptive immunological responses. DAMPs activate pattern recognition receptors (PRRs), including Toll-like receptors (TLRs), which mediate immunogenic effects (3). Nucleic acids (including mRNA) are danger-associated molecular patterns (DAMPs) that activate pattern recognition receptors (PRRs), including Toll-like receptors (TLRs), which mediate immunogenic effects. As a result, the COVID-19 mRNA vaccines now available do not require adjuvants (4). If human strains are employed, the most widely used adenovirus platforms are vulnerable to variable prevalence of pre-existing anti-vector immunity in the population (2). They have, however, been shown to be successful in producing a long-lasting response and do not require additional adjuvants; in fact, some of them are designed for a single-dose regimen (e.g., Ad26.COV2.S and Ad5-nCoV). Because the vector virus typically exclusively infects chimps, non-human adenovirus strains are immune to pre-existing host immunity (e.g., AZD1222). Adjuvants, on the other hand, need special consideration when it comes to vaccine-related skin toxicity since they have the potential to cause off-target inflammatory responses (5). After all, vaccine-related adverse effects frequently affect the skin (6). This is an expected finding, given that viral infections can cause cutaneous eruptions (e.g., measles-morbillivirus) or paraviral cutaneous reactions (erythema multiforme-herpes simplex virus; Gianotti-Crosti syndrome-hepatitis B virus and others; papular pruritic gloves and socks syndrome-parvovirus B19 and others) (7). In a small number of individuals, SARS-CoV-2 infections can cause vesicular, urticarial, maculopapular, and chilblain-like eruptions $(8,9)$. Similar pathophysiological responses might be detected after an immunogenic challenge with a comparable vaccination. Finally, cutaneous adverse drug reactions (ADRs) appear to be common with the administration of COVID-19 vaccinations, and include erythema, swelling, itching, pernio-like lesions, and widespread rashes (10). Despite the fact that they might be frightening for patients and treating physicians, most clinical studies do not accurately reflect them from a dermatological standpoint. Erythema, swelling, itch, or nodules, for example, are sometimes referred to as "local responses," whereas eczematous, vesicular, and morbilliform reactions are frequently referred to as "rash". Furthermore, several more recent prospective "real-world" investigations on ADRs to COVID-19 vaccinations included responses within the first week or even only the first three days following immunization (11). Dermatologists have only lately begun to describe cutaneous ADRs with sufficient distinction to demonstrate that vaccinations can cause a variety of inflammatory skin responses $(10,12)$. It's also worth noting that the time between immunization and the beginning of delayed skin responses might be as long as 10 days (13). Furthermore, individuals with pre-existing diseases needing immunosuppressive treatment are often excluded from clinical trials; consequently, flares of pre-existing chronic inflammatory dermatoses may have been underreported so far (14).

In this systematic review, we want to draw attention to the wide range of possible cutaneous inflammatory responses that might occur during vaccination administration in order to help healthcare providers better understand these manifestations. This systematic review aims to summarize and integrate the findings of studies regarding cutaneous side effects of COVID-19 vaccines.

\section{Methods}

2.1. Study design 
This study was a systematic review that was performed by searching the scientific databases of PubMed, Scopus, Science direct, and Web of knowledge for relevant English articles published from the beginning of the COVID-19 to10/5/2021. Analysis of retrieved articles was performed in four-step selection process of identification, screening, eligibility, and inclusion criteria, based on Preferred Reporting Items for Systematic Reviews and Meta-Analyses (PRISMA) checklist.

\subsection{Search strategy}

We conducted a comprehensive search on the scientific databases using MeSH keywords including:

COVID-19 OR SARS-CoV-2 OR Coronavirus OR "Corona virus" OR COVID; AND vaccine OR vaccines OR vaccination OR sputnik OR astrazeneca OR Pfizer OR sinopharm OR moderna OR bharat OR "Johnson \&Johnson"; AND effect OR reaction OR adverse OR subsequence OR consequence OR complication OR outcome OR aftereffect OR disorder OR disturbance OR sequel; AND skin OR cutaneous OR Derm OR dermis OR keratinocyte OR dermatology.

To find more studies, the references in the relevant papers were also followed up. The search was performed by two independent researchers.

\subsection{Study selection}

In the first step two researchers reviewed the retrieved articles and removed the duplicates. In other steps, the researchers screened the title and abstract of the records and the ineligible studies were removed. Then, the authors surveyed the full-text of the remaining studies based on inclusion and exclusion criteria and the eligible studies (case report, case series, and cross-sectional) were identified.

We excluded the articles which were topic to at least one of the following criteria:

- Non-original articles including reviews, protocols and editorials.

- Abstract papers, articles without obtainable full texts, and conference abstracts.

- Clinical trials which were in progress without yet published results.

- No COVID-19 vaccine trials articles.

- Non-English articles.

\subsection{Data extraction}

The following information was extracted from full-text articles by three authors: first author (reference); type of study; country of study; population; medical history; type of vaccine; cutaneous manifestations; duration of cutaneous manifestations; mechanism of cutaneous reactions; diagnostic assessment; management; outcomes; and other findings and transferred into a data spread table. These specifications and the corresponding table were designed by three members of our team. In order to eradicate any probable duplications, the selected articles were investigated by other researchers once again.

\subsection{Quality assessment}

As mentioned formerly, we utilized the Preferred Reporting Items for Systematic Reviews and Meta-Analyses (PRISMA) checklist for evaluation and analyze of articles. The quality and relevance of the articles were investigated by two independent and experienced members. In any case their decisions differed, a third independent researcher confirmed their disagreements.

Table 1-Characteristics of included studies 


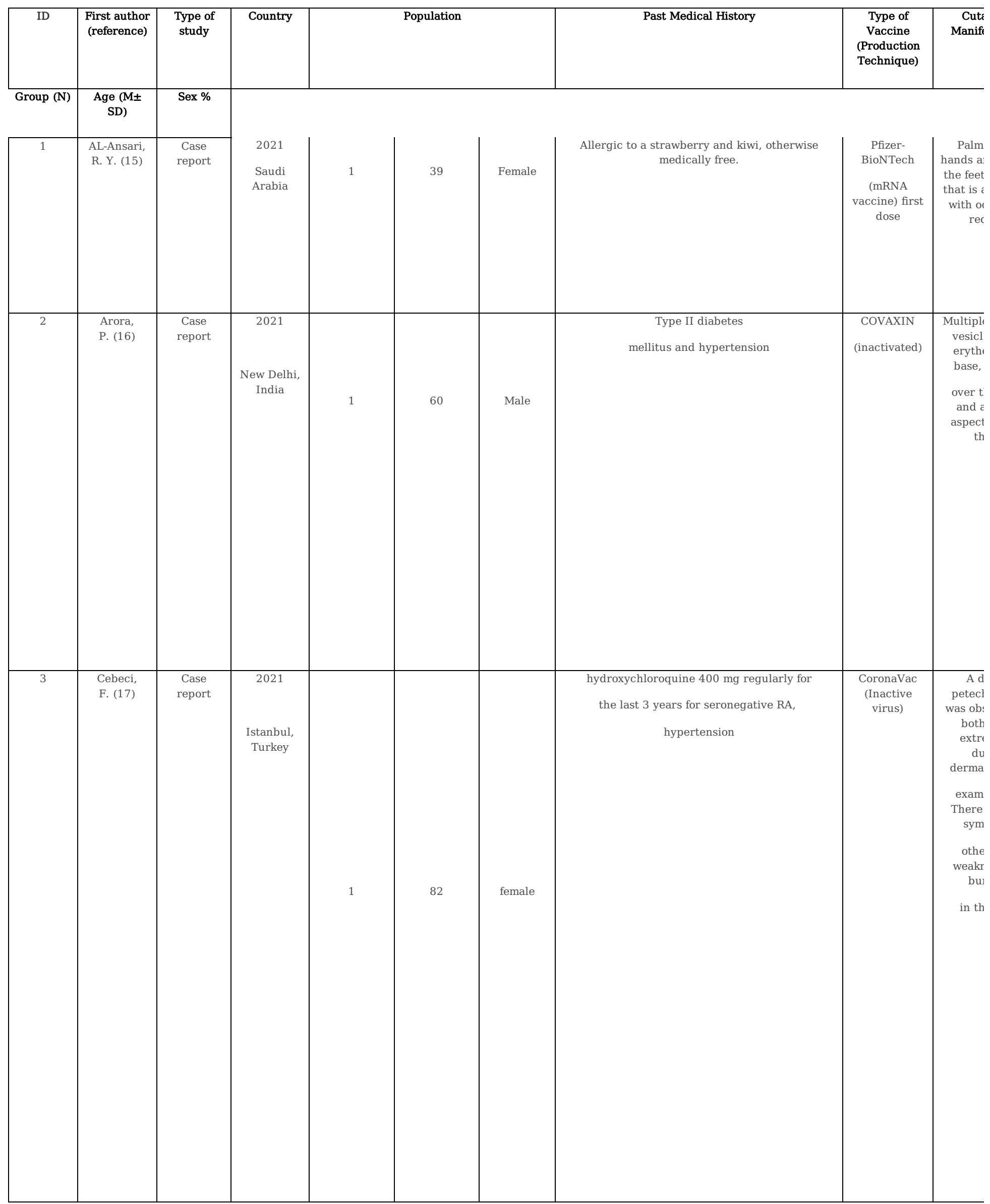




\begin{tabular}{|c|c|c|c|c|c|c|c|c|c|}
\hline ID & $\begin{array}{c}\text { First author } \\
\text { (reference) }\end{array}$ & $\begin{array}{l}\text { Type of } \\
\text { study }\end{array}$ & Country & & Population & & Past Medical History & $\begin{array}{l}\text { Type of } \\
\text { Vaccine } \\
\text { (Production } \\
\text { Technique) }\end{array}$ & $\begin{array}{r}\text { Cuti } \\
\text { Manifi }\end{array}$ \\
\hline 4 & $\begin{array}{c}\text { Farinazzo, } \\
\text { E. (18) }\end{array}$ & Case series & $\begin{array}{l}2021 \\
\text { North east } \\
\text { Italy }\end{array}$ & 46 & $\begin{array}{l}\text { Mean:44.39 } \\
\text { SD: } 12.36 \\
(18-31]: 6 \\
(31-44]: 16 \\
(44-57]: 17 \\
(57-70): 7\end{array}$ & $\begin{array}{c}\text { Female:41 } \\
\text { (89\%) } \\
\\
\text { Male: } 5 \\
(11 \%)\end{array}$ & - & $\begin{array}{c}\text { BioNTech/ } \\
\text { Pfizer vaccine } \\
\\
\text { (mRNA } \\
\text { based) }\end{array}$ & $\begin{array}{r}\text { Male: } \\
40 \% \\
\text { urtica } \\
\text { Pityria } \\
\text { like ras } \\
\text { neck } \\
\text { limb } \\
\text { trur } \\
\text { Erythe } \\
\text { inocule } \\
2 \\
\text { Fes } \\
\text { Itchin } \\
\text { Urtica } \\
19 \% \mathrm{E} \\
31 \% \varsigma \\
14 \% \text { Mc } \\
\text { eruptior } \\
\text { drug er } \\
\text { Chilblais } \\
2 \% \text { Pj } \\
\text { rosea- } \\
2 \\
\text { Malar } \\
\text { painfu } \\
\subseteq \\
\text { Herpes } \\
\text { chest } \\
\text { rasl } \\
\text { chilb] } \\
\text { manifes } \\
\text { the first } \\
\text { finger c } \\
2 \% . \\
\text { eryth } \\
\text { erythı } \\
\text { macule } \\
\text { the han } \\
\text { drug } \\
10 \% \text { re } \\
\text { rity }\end{array}$ \\
\hline 5 & $\begin{array}{c}\text { Hoff, N. P. } \\
\text { (19) }\end{array}$ & Case series & $\begin{array}{c}2021 \\
\text { Germany }\end{array}$ & 11 & $50.09 \pm 13.18$ & $\begin{array}{l}\text { Male: } \\
2 \\
(18 \%) \text {, } \\
\text { female: } \\
9(82 \%)\end{array}$ & $\begin{array}{l}\text { Patient no. } 7 \text { (female } 44 \text { ) had obesity as a } \\
\text { comorbidity factor }\end{array}$ & $\begin{array}{c}\text { Moderna } \\
\text { (mRNA-1273) } \\
\text { vaccine }\end{array}$ & 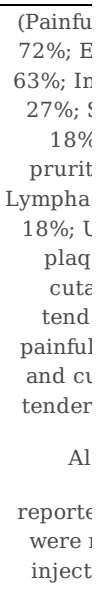 \\
\hline
\end{tabular}




\begin{tabular}{|c|c|c|c|c|c|c|c|c|c|}
\hline ID & $\begin{array}{c}\text { First author } \\
\text { (reference) }\end{array}$ & $\begin{array}{l}\text { Type of } \\
\text { study }\end{array}$ & Country & \multicolumn{3}{|c|}{ Population } & Past Medical History & $\begin{array}{l}\text { Type of } \\
\text { Vaccine } \\
\text { (Production } \\
\text { Technique) }\end{array}$ & $\begin{array}{r}\text { Cut: } \\
\text { Manifi }\end{array}$ \\
\hline 6 & $\begin{array}{c}\text { Hussain, } \mathrm{K} \text {. } \\
(20)\end{array}$ & $\begin{array}{l}\text { Case } \\
\text { report }\end{array}$ & $\begin{array}{c}2021 \\
\text { London, UK }\end{array}$ & 1 & 62 & 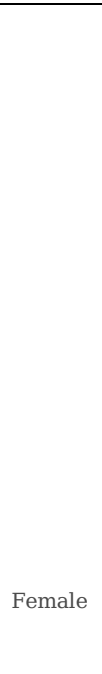 & $\begin{array}{c}\text { Metastatic melanoma and } \\
\text { related CPI therapy } \\
\text { (Nivolumab and ipilimumab) myocarditis. new } \\
\text { onset of } \\
\text { symptoms consistent with Raynaud disease (RD) }\end{array}$ & $\begin{array}{c}\text { Pfizer } \\
\text { (BioNTech- } \\
\text { COVID-19 } \\
\text { RNA vaccine) }\end{array}$ & $\begin{array}{r}\text { Mu } \\
\text { men } \\
\text { involven } \\
\text { gra } \\
\text { erythr } \\
\text { with sı } \\
\text { blisteri } \\
\text { on the } \\
\text { thigh a } \\
\end{array}$ \\
\hline 7 & $\begin{array}{l}\text { Català, A. } \\
\text { (21) }\end{array}$ & $\begin{array}{c}\text { cross- } \\
\text { sectional }\end{array}$ & $\begin{array}{l}\text { Spain } \\
2021\end{array}$ & 405 & $50.7(17.6)$ & $\begin{array}{l}\text { Female } \\
325 \\
(80.2 \%) \\
\text { Male } 80 \\
(19.8 \%)\end{array}$ & $\begin{array}{c}\text { Atopic dermatitis } 28 \text { (6.9); Allergic asthma } 24 \\
\text { (5.9); Allergic rhinitis } 42(10.4) \text {; Urticaria } 26 \\
\text { (6.4); History of allergy to drugs or excipients } \\
47 \text { (11.6) }\end{array}$ & $\begin{array}{c}\text { (Pfizer- } \\
\text { BioNTech), } \\
\text { mRNA-1273 } \\
\text { (Moderna), } \\
\text { AZD1222 } \\
\text { (AstraZeneca) }\end{array}$ & $\begin{array}{r}\text { Covid-A } \\
\text { HSV re: } \\
3.7 \% \\
\text { react } \\
10.1 \% \\
\text { vesicu] } \\
\text { Pityria } \\
\text { like } \\
\text { Morb } \\
8.9 \% \text {; } \\
\text { Angi } \\
14.6 \% ;\end{array}$ \\
\hline
\end{tabular}




\begin{tabular}{|c|c|c|c|c|c|c|c|c|c|}
\hline ID & $\begin{array}{l}\text { First author } \\
\text { (reference) }\end{array}$ & $\begin{array}{l}\text { Type of } \\
\text { study }\end{array}$ & Country & & Population & & Past Medical History & $\begin{array}{c}\text { Type of } \\
\text { Vaccine } \\
\text { (Production } \\
\text { Technique) }\end{array}$ & $\begin{array}{r}\text { Cuti } \\
\text { Manifi }\end{array}$ \\
\hline 8 & $\begin{array}{c}\text { Juárez } \\
\text { Guerrero, } \\
\text { A. (22) }\end{array}$ & Case series & Spain 2021 & 26 & $45 \pm 4.66$ & $\begin{array}{c}\text { Female: } 25 \\
(96.2) \\
\text { male } \\
: 1(3.8)\end{array}$ & $\begin{array}{l}\text { Previous history of } 1 \text { or more of the following: } 9 \\
(60 \%) \text { rhinoconjunctivitis, } 10(66.7 \%) \text { asthma, } 5 \\
(33.3 \%) \text { anaphylaxis (latex, kiwi and } \\
\text { Hymenoptera, arylpropionics, contrast media), } 2 \\
(13.3 \%) \text { chronic urticaria, and } 1 \text { (6.7\%) nickel } \\
\text { and } \\
\text { methylchloroisothiazolinone/methylisothiazolinone } \\
\text { allergic contact dermatitis. }\end{array}$ & $\begin{array}{l}\text { Pfizer- } \\
\text { BioNTech, } \\
19(27 \%) \\
\\
\text { Moderna } \\
7(73 \%)\end{array}$ & $\begin{array}{r}\text { DLLR or } \\
13 \text { of } 2: \\
\text { (57\%) } \\
\text { these 2: } \\
(4: \\
\text { expes } \\
\text { delay } \\
\text { reaction } \\
\text { from ] } \\
\text { deve } \\
\text { gene } \\
\text { maculı } \\
\text { exant } \\
\text { exfoliati } \\
\text { skin of t } \\
1 \text { c } \\
\text { gene } \\
\text { exanth } \\
\text { pust } \\
\text { (AG } \\
\text { gene } \\
\text { micro } \\
\text { exan } \\
\text { accompi } \\
7 \text {-cm } \mathrm{t} \\
\text { the shol } \\
1 \text { multi } \\
\text { drug e } \\
\text { (M] }\end{array}$ \\
\hline 9 & $\begin{array}{c}\text { Larson, V. } \\
\text { (23) }\end{array}$ & Case series & $\begin{array}{l}\text { USA } \\
2021\end{array}$ & 12 & $69.25 \pm 19.5$ & $\begin{array}{c}\text { Female } \\
50 \%, \\
\text { male } \\
50 \%\end{array}$ & $\begin{array}{c}\text { All items are } 8.3 \% \\
\text { Lichen simplex chronicus, } \\
\text { Inflammatory bowel disease (on mesalamine, } \\
\text { vedolizumab) } \\
\text { eczema, } \\
\text { Acne vulgaris and herpes simplex virus, } \\
\text { and allergic rhinitis, } \\
\text { psoriasis, } \\
\text { childhood atopic dermatitis }\end{array}$ & $\begin{array}{c}\text { Pfizer } \\
\text { BioNTech=5 } \\
\text { Moderna=7 }\end{array}$ & $\begin{array}{r}\mathrm{COV}] \\
16 \\
\text { Erupt } \\
\text { Ecze } \\
\text { patche } \\
\text { Pityria } \\
\text { like e } \\
8.3 \% ; \\
\text { purpuri } \\
8 . \\
\text { Eryther } \\
\text { eder } \\
\text { plaqu } \\
\text { Bu } \\
\text { pemi } \\
1 €\end{array}$ \\
\hline 10 & $\begin{array}{c}\text { Lopatynsky- } \\
\text { Reyes, E. Z. } \\
(24)\end{array}$ & Case series & $\begin{array}{c}\text { Mexico } \\
2021\end{array}$ & $\begin{array}{c}2 \\
\text { International } \\
\text { Healthcare } \\
\text { Workers }\end{array}$ & $\begin{array}{l}31, \\
28\end{array}$ & $\begin{array}{c}\text { Female } \\
50 \% \\
\text { male } \\
50 \%\end{array}$ & $\mathrm{~N} / \mathrm{A}$ & $\begin{array}{l}\text { Pfizer- } \\
\text { (BioNTech), } \\
\text { Moderna } \\
\text { (Both second } \\
\text { dose) }\end{array}$ & $\begin{array}{r}\text { inflamr } \\
\text { the } \mathrm{B}( \\
\text { erythı } \\
\text { rea } \\
\text { indu] } \\
\text { mild }\end{array}$ \\
\hline
\end{tabular}




\begin{tabular}{|c|c|c|c|c|c|c|c|c|c|}
\hline ID & $\begin{array}{c}\text { First author } \\
\text { (reference) }\end{array}$ & $\begin{array}{l}\text { Type of } \\
\text { study }\end{array}$ & Country & & Population & & Past Medical History & $\begin{array}{c}\text { Type of } \\
\text { Vaccine } \\
\text { (Production } \\
\text { Technique) }\end{array}$ & $\begin{array}{r}\text { Cuti } \\
\text { Manifi }\end{array}$ \\
\hline 11 & $\begin{array}{l}\text { McMahon, } \\
\text { D. E. } \\
\text { (7) }\end{array}$ & $\begin{array}{l}\text { Cross } \\
\text { Sectional } \\
\text { (Registry- } \\
\text { based } \\
\text { study) }\end{array}$ & $\begin{array}{l}\text { USA } \\
2021\end{array}$ & 414 & $\begin{array}{c}44 \\
(36-59)\end{array}$ & $\begin{array}{l}\text { Female90\% } \\
\text { Male: } 10 \%\end{array}$ & $\begin{array}{c}\text { Atopic dermatitis } 84 \% \text {; contact dermatitis } 2.9 \% \text {; } \\
\text { psoriasis } 2.2 \% \text {; urticaria } 1.7 \% \text {; Acne vulgaris } \\
1.4 \% \text {; } \\
\text { Hypertension } 15 \% \text {; Obstructive lung disease } 4.8 \% \text {; } \\
\text { morbid obesity } 4.1 \% \text {; cardiovascular disease } \\
\text { 2.4\%; diabetes mellitus } 3.6 \% \text {; Rheumatologic } \\
\text { disease } 2.4 \% \text {; malignancy } 1.9 \%\end{array}$ & $\begin{array}{l}\text { Moderna 83\% } \\
\text { Pfizer 17\% }\end{array}$ & 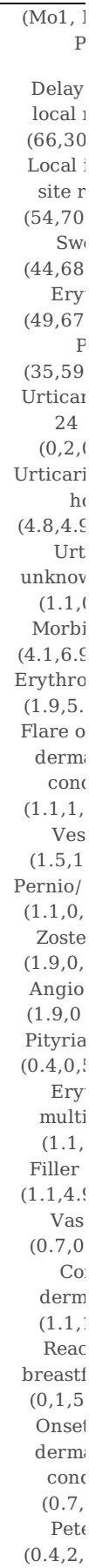 \\
\hline 12 & $\begin{array}{l}\text { Peigottu, } \\
\text { M. F. (25) }\end{array}$ & Case series & $\begin{array}{l}\text { Italy } \\
2021\end{array}$ & 9 & $46 \pm 9.26$ & \begin{tabular}{|}
$\begin{array}{r}\text { Female } \\
88.9 \% \\
\text { male } \\
11.1 \%\end{array}$ \\
\end{tabular} & $\begin{array}{c}\begin{array}{c}\text { Significant allergology history } 100 \% ; \text { Atopy, drug } \\
\text { allergy: } 88.8 \% ; \\
\text { Previous angioedema: } \\
11.2 \%\end{array} \\
\end{array}$ & $\begin{array}{c}\text { Pfizer } \\
\text { (BioNTech) }\end{array}$ & $\begin{array}{r}\text { Wide } \\
\text { pruritr } \\
\text { facial er } \\
\text { macul, } \\
\text { ra: } \\
\text { Glottis } \\
\text { eryth } \\
\text { edema } \\
\text { maculc } \\
\text { lesi } \\
\text { Urtica } \\
\text { with art }\end{array}$ \\
\hline
\end{tabular}




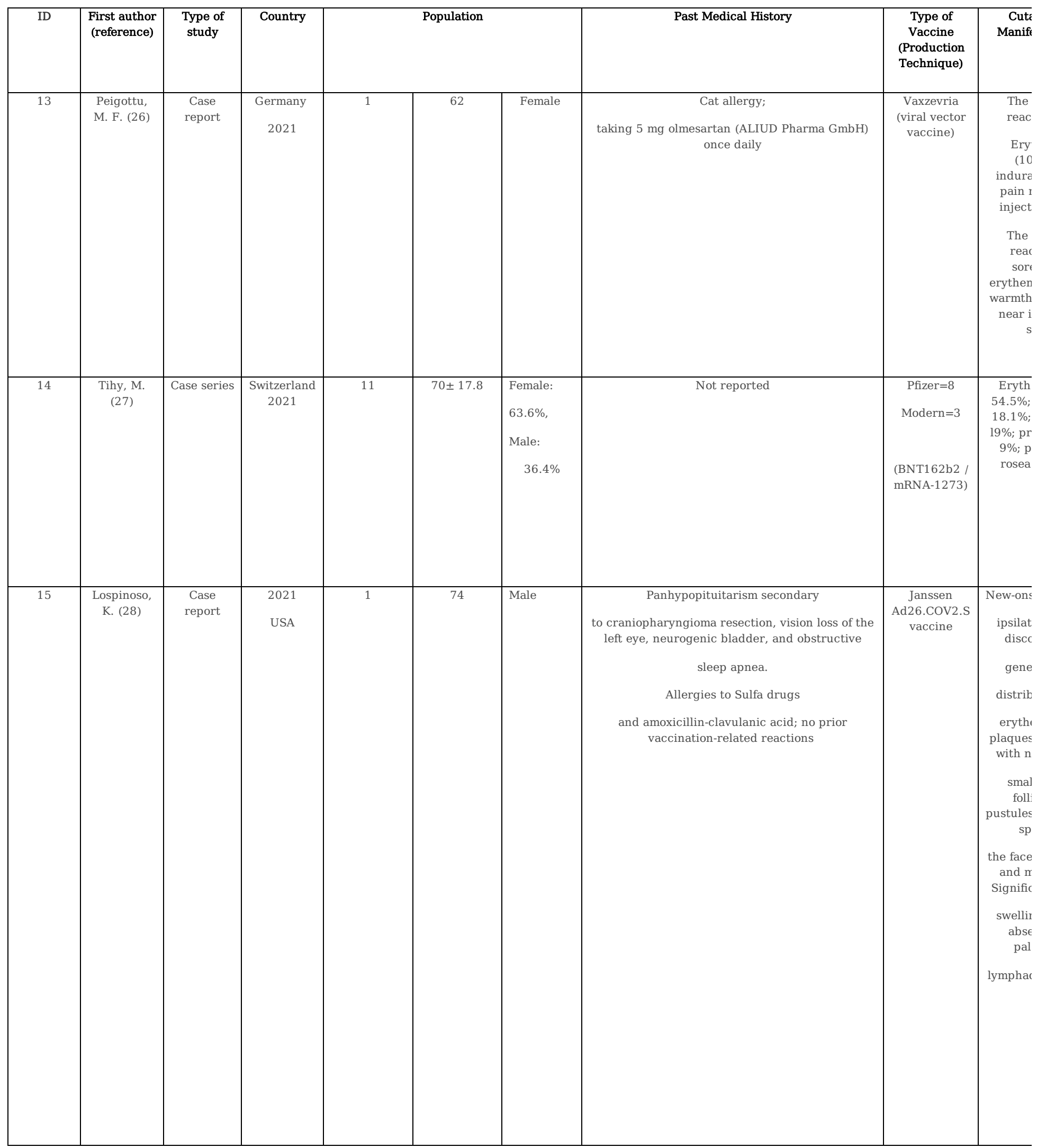




\begin{tabular}{|c|c|c|c|c|c|c|c|c|c|}
\hline $\begin{array}{l}\text { ID } \\
16\end{array}$ & \multirow{2}{*}{$\begin{array}{c}\begin{array}{c}\text { First author } \\
\text { (reference) }\end{array} \\
\text { Eid, } \\
\text { Edward } \\
(29)\end{array}$} & \multirow{2}{*}{$\begin{array}{c}\begin{array}{c}\text { Type of } \\
\text { study }\end{array} \\
\\
\text { Case } \\
\text { report }\end{array}$} & \multirow{2}{*}{$\begin{array}{c}\text { Country } \\
\\
2021 \\
\text { Lebanon }\end{array}$} & \multicolumn{3}{|c|}{ Population } & \multirow{2}{*}{$\begin{array}{c}\text { Past Medical History } \\
\text { Hypertension, coronary artery } \\
\begin{array}{c}\text { disease, and antineutrophilic cytoplasmic } \\
\text { antibodyrelated glomerulonephritis }\end{array}\end{array}$} & \multirow{2}{*}{ 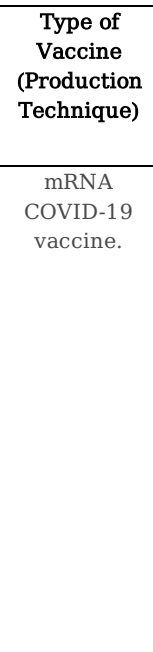 } & \multirow{2}{*}{ 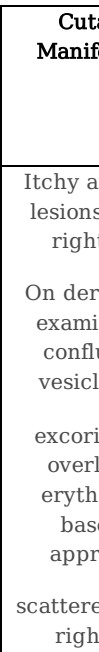 } \\
\hline 16 & & & & 1 & 79 & Male & & & \\
\hline 17 & $\begin{array}{c}\text { Chopra, S. } \\
\text { (30) }\end{array}$ & $\begin{array}{l}\text { Case } \\
\text { report }\end{array}$ & $\begin{array}{c}2021 \\
\text { USA }\end{array}$ & 1 & 56 & Female & - & Moderna & $\begin{array}{r}\text { intensel } \\
\text { rash ol } \\
\mathrm{h} \\
\text { and spr } \\
\text { left elb } \\
\text { hands, } \\
\text { feet, } \\
\text { violace } \\
\text { on th } \\
\text { finge } \\
\text { hand; er } \\
\text { viole } \\
\text { papul } \\
\text { palm } \\
\text { hanı } \\
\text { dorsal } \\
\text { urticari } \\
\text { on th } \\
\text { aspec } \\
\text { hands, } \\
\text { and upp } \\
\text { of tl } \\
\text { occasior } \\
\text { day a } \\
\text { vacci }\end{array}$ \\
\hline
\end{tabular}

ACD, allergic contact dermatitis; AGEP, acute generalized exanthematous pustulosis; AH1, antihistaminics H1; CU, chronic urticaria; DLLR, delayed large local reaction; MFDE, multiple fixed drug eruptions; MPE, maculopapular exanthema; N-ERD, NSAID-exacerbated respiratory disease; OCS, oral corticosteroids; RC, rhinoconjunctivitis; TCS, topical corticosteroids; DHR, dermal hypersensitivity reaction; CRP, C reactive protein; DIF, direct immunofluorescence.

\section{Results}

A total of 69 potentially relevant articles were identified through the initial searches. After removing duplicates 58 articles remained, and then 32 articles were excluded by screening titles and abstracts, and the full texts of 26 remaining articles were assessed for eligibility. Finally, 17 studies met the inclusion criteria and were included in this systematic review as Figure 1 shows.

The studies and clinical characteristics are summarized in Table 1. These studies were conducted in different countries, eight case reports from Saudi Arabia, India, Turkey, and United Kingdom, as well as seven case series from North east Italy, and Germany and 2 cross-sectional studies.

Our results showed that injection site reactions and delayed large local reactions were the most common side effects arising from all vaccine types followed by: itchiness, urticarial rash on the neck, upper limbs, and trunk, morbilliform eruptions, erythema at the inoculation site, swelling, etc. $(7,18,19)$.

Most patients showed cutaneous reactions after the first dose of vaccination $(7,18,19,22,23)$. Some patients had very rare adverse dermatological reactions after first and second doses $(7,18,23)$. The Majority of adverse reactions were reported after mRNA-based vaccines $(15,18-20)$.

The onset and duration of dermatological manifestations were ranged from 1-21 days after vaccination except for three reports that reactions appeared within the first hours $(16,17,20)$. Moreover, delayed cutaneous ADRs may occur after several days, either as a primary manifestation or as a flare of a pre-existing inflammatory dermatosis (28). 
The cutaneous side effects of the covid-19 vaccines appear to be more common in women $(7,15,17-22,25,27)$ and diverse age groups, however, most were middle-aged $(7,18,19,21,22,25)$.

None of the skin reactions after the first dose of the vaccine prohibited the administration of the second dose except for six subjects that were advised not applying the second dose $(18,25)$. because it is currently unclear whether it should be regarded as a risk factor for anaphylaxis, although the timing of onset after exposure is not consistent with a type I lg-E mediated reaction (18). Besides, 18 individuals did not plan to receive the second dose (7, 22, 23). No longterm cutaneous sequelae remain in any of the affected persons (19).

Most of the encountered skin reactions were self-limiting, and need little or no therapeutic intervention $(26,27,31)$. Some patients treated conservatively by anti-histamine $(7,15,19,21-23,25)$, topical glucocorticoids $(7,19,21-23,28,30)$, prednisolone $(21,23,25,28)$, antibiotic $(21,23)$, systemic antiviral $(21,29)$, and intravenous methylprednisolone (20).

Investigating mechanisms of adverse cutaneous reactions, it was suggested that type I allergic reactions occur due to dimerization of high-affinity IgE receptors in sensitized individuals after contact with an allergen (e.g., PEGs such as Polyethylene glycol-2000 (PEG-2000), an excipient of the vaccine, may play a role in the allergic reactions after vaccination $(7,18,23,26)$.

\section{Discussion}

We entered a new stage of the COVID-19 pandemic in 2021. Given that mass vaccinations are administering across the world and more vaccines have been approved, diagnose the cutaneous side effects of those is important. Understanding the cutaneous demonstrations of COVID-19 vaccines is a key factor for giving proper guidance for the vaccine. The current systematic review summarized and integrated the findings of studies regarding cutaneous side effects due to COVID-19 vaccines.

Firstly, according to our findings, it is noteworthy to note that severe cutaneous side effects are very rare and approved vaccines have satisfactory safety profiles. Most cutaneous reactions are self-limiting and need little or no treatment. In addition, the findings of this review showed injection site reactions are prevalent cutaneous side effects due to COVID-19 vaccines. COVID-19 vaccines like other vaccines such as the combined pneumococcal vaccine often cause cutaneous reactions near the injection site including erythema and swelling (32) and are because of non-specific stimulation of inflammation (12). Consistent with our findings, Sun et al.'s study found that early-onset local injection reactions including erythema, pruritus, swelling, and induration are the most frequent cutaneous side effects with COVID-19 vaccines (33). Another study reported that pain and erythema are the most frequent cutaneous side effects of all COVID-19 vaccines (31). Furthermore, a delayed large local reaction also referred to as "COVID arm", which is characterized by erythematous and edematous patch at the local of injection onset at least 4 days or more after vaccination, was reported mainly in mRNA-based vaccines $(7,13,34)$. Although these reactions are self-limiting, topical steroids or oral medications can be applied to alleviate these reactions (33). Clinicopathological classification can be helpful in the early diagnosis and management of the dermal reactions to mRNA COVID-19 vaccines (27).

Our review findings revealed the cutaneous side effects of COVID-19 vaccines were mostly affected middle-age populations. Similarly, current evidence shows that local injection site reactions are more seen in the younger population compared the older people $(35,36)$. Moreover, according to our findings, the most onset of cutaneous manifestation was 1-21 days after vaccination. A systematic review reported that the most frequent adverse cutaneous reactions occurred within 7 days after injection (33). Our review focused only on cutaneous side effects of COVID-19 vaccines in real-world settings, but the mentioned systematic review included studies both from vaccines trial and real-world settings that may explain this inconsistent finding.

The present review showed that the cutaneous side effects of the covid-19 vaccines appear to be more common in women. The cause is may due to several probable factors. Women show more vigorous immune responses to external antigens than men. Accordingly, numerous research has been shown that women have more immune responses to vaccines and also experience more side effects $(37,38)$. However, the high prevalence of side effects of COVID-19 vaccines among women may reflect reporting bias. Because women constitute the vast majority of the healthcare workforce who were the first group to be vaccinated against COVID-19. In addition, the healthcare workforce is more likely to be visited by physicians (33). However, there is no definite identified reason for this issue and requires to be addressed in forthcoming studies.

According to our review findings, after administering the first dose of the COVID-19 vaccine, only six persons were given the advice do not to apply the second dose due to diffuse urticaria and maculopapular rash. It is currently unclear whether these after the first dose of the COVID-19 vaccine should be considered as a potential risk factor for anaphylaxis reactions (39). However, it seems that people who have presented potentially anaphylactic reactions such as diffuse urticaria to the first dose of a COVID-19 vaccine require to be referred to an allergist for accurate assessment (40).

The mechanism of possible allergic reactions related to COVID-19 vaccines has not yet been discovered, and any possible allergens have not yet been recognized. However, our review showed that the majority of cutaneous adverse reactions are associated with mRNA-based vaccines, which is consistent with a previous study (33). Among the various excipients in COVID-19 vaccines, it seems that polyethylene glycol (PEG) in mRNA vaccines is one of the suspected causes of allergic reactions (12). Nevertheless, additional work is required to further dissect the phenomenon and reveal the underlying immunologic mechanism and determine the best suitable vaccine type for individual groups of patients.

\section{Limitations}

The current review presents findings on an evolving universal phenomenon and some limitations should be considered. The studies included in this review were mainly case reports and case series, which can make their results challenging. Therefore, future studies with robust designs such as cohorts in this field are needed to provide more accurate findings with more details. In addition, due to potential underreporting of complications, side effects such as cutaneous 
reactions following COVID-19 vaccination may be more than reported. More clinical trials still are needed to investigate the side effect profile of all COVID-19 vaccines.

\section{Conclusions}

Cutaneous side effects and allergic reaction is one of the expected complications after any COVID-19 or non-COVID-19 vaccination. Recognizing these reactions can have a key role in vaccine strategy because anxieties about developing reactions can considerably influence people's willingness to receive the first dose or return for a second dose. Injection site reactions are one of the most common cutaneous side effects after COVID-19 vaccines, which most of these reactions are mild or moderate, have no serious consequences, and are usually self-limiting without any interventions. Therefore, these reactions should not discourage people from vaccination.

It seems that cutaneous side effects with mRNA-based COVID-19 vaccines are more common; however, the COVID-19 vaccine boundary is growing rapidly and future research results may provide more detailed information. In certain groups such as patients with allergies and a history of local injection reactions, it seems that pre-vaccination counseling and giving guidance and use of antihistamines and topical medications may benefit.

\section{Declarations}

\section{Ethics approval and consent to participate}

This study was extracted from the study project with code IR.ESFARAYENUMS.REC.1400.004 entitled "Cutaneous adverse reactions of COVID-19 vaccines: a systematic review" conducted at Esfarayen University of Medical Science in 2021.

\section{Ethical Consideration}

This article is based on published data, and hence ethical approval is not required.

Availability of data and material: The authors expressed that all information provided in this article can be shared.

Competing interests: The all authors declare that there is no conflict of interest regarding the publication of this article.

Funding: This study received grant from Esfarayen University of Medical Science.

\section{Authors' contributions:}

1. The conception and design of the study: Ahmadreza Shamsabadi and Kowsar Qaderi

2. Acquisition of data: Mohammad Hossein Golezar and Bagher Moradi

3. Analysis and interpretation of data: Abbas Mardani

4. Drafting the article: Manthar Ali Mallah

5. Revising it critically for important intellectual content: Samira Golezar

6. Final approval of the version to be submitted: All authors

\section{References}

1. Kaur SP, Gupta V. COVID-19 Vaccine: A comprehensive status report. Virus research. 2020;288:198114.

2. Jeyanathan M, Afkhami S, Smaill F, Miller MS, Lichty BD, Xing Z. Immunological considerations for CoVID-19 vaccine strategies. Nature reviews Immunology. 2020;20(10):615-32.

3. Preissner KT, Fischer S, Deindl E. Extracellular RNA as a versatile DAMP and alarm signal that influences leukocyte recruitment in inflammation and infection. Frontiers in Cell and Developmental Biology. 2020;8:1618.

4. Pascolo S. Synthetic messenger RNA-based vaccines: from scorn to hype. Viruses. 2021;13(2):270.

5. He Q, Mao Q, Zhang J, Bian L, Gao F, Wang J, et al. COVID-19 Vaccines: Current Understanding on Immunogenicity, Safety, and Further Considerations. Frontiers in Immunology. 2021;12.

6. Rosenblatt AE, Stein SL. Cutaneous reactions to vaccinations. Clinics in dermatology. 2015;33(3):327-32.

7. McMahon DE, Amerson E, Rosenbach M, Lipoff JB, Moustafa D, Tyagi A, et al. Cutaneous reactions reported after Moderna and Pfizer COVID-19 vaccination: A registry-based study of 414 cases. Journal of the American Academy of Dermatology. 2021;85(1):46-55.

8. Galván Casas C, Catala A, Carretero Hernández G, Rodríguez-Jiménez P, Fernández-Nieto D, Rodríguez-Villa Lario A, et al. Classification of the cutaneous manifestations of COVID-19: a rapid prospective nationwide consensus study in Spain with 375 cases. British Journal of Dermatology. 2020;183(1):717.

9. Novak N, Peng W, Naegeli MC, Galvan C, Kolm-Djamei I, Brüggen C, et al. SARS-CoV-2, COVID-19, skin and immunology-What do we know so far? Allergy. 2021;76(3):698-713.

10. McMahon DE, Amerson E, Rosenbach M, Lipoff JB, Moustafa D, Tyagi A, et al. Cutaneous reactions reported after Moderna and Pfizer CoVID-19 vaccination: A registry-based study of 414 cases. Journal of the American Academy of Dermatology. 2021;85(1):46-55. 
11. Bae S, Lee YW, Lim SY, Lee JH, Lim JS, Lee S, et al. Adverse Reactions Following the First Dose of ChAdOx1 nCoV-19 Vaccine and BNT162b2 Vaccine for Healthcare Workers in South Korea. Journal of Korean medical science. 2021;36(17):e115.

12. Bogdanov G, Bogdanov I, Kazandjieva J, Tsankov N. Cutaneous adverse effects of the available COVID-19 vaccines. Clinics in Dermatology. 2021.

13. Blumenthal KG, Freeman EE, Saff RR, Robinson LB, Wolfson AR, Foreman RK, et al. Delayed large local reactions to mRNA-1273 vaccine against SARSCoV-2. New England Journal of Medicine. 2021;384(13):1273-7.

14. Niebel D, Ralser-Isselstein V, Jaschke K, Braegelmann C, Bieber T, Wenzel J. Exacerbation of subacute cutaneous lupus erythematosus following vaccination with BNT162b2 mRNA vaccine. Dermatologic Therapy. 2021.

15. Al-Ansari RY, Al-Sharari M, Al-Saadi T. Palms and soles itchiness as a side effect of COVID-19 vaccination. Journal of infection and public health. 2021.

16. Arora P, Sardana K, Mathachan SR, Malhotra P. Herpes zoster after inactivated COVID-19 vaccine: A cutaneous adverse effect of the vaccine. J Cosmet Dermatol. 2021.

17. Cebeci F, Kartal İ. Petechial skin rash associated with CoronaVac vaccination: first cutaneous side effect report before phase 3 results. European journal of hospital pharmacy: science and practice. 2021.

18. Farinazzo E, Ponis G, Zelin E, Errichetti E, Stinco G, Pinzani C, et al. Cutaneous adverse reactions after m-RNA COVID-19 vaccine: early reports from Northeast Italy. J Eur Acad Dermatol Venereol. 2021;35(9):e548-e51.

19. Hoff NP, Freise NF, Schmidt AG, Firouzi-Memarpuri P, Reifenberger J, Luedde T, et al. Delayed skin reaction after mRNA-1273 vaccine against SARS-CoV-2: a rare clinical reaction. European journal of medical research. 2021;26(1):98.

20. Hussain K, Kawsar A, Weir J, Au L, Turajlic S, Larkin J, et al. Severe cutaneous adverse reaction following COVID-19 vaccination and immunotherapy: a second hit? Clinical and Experimental Dermatology.

21. Català A, Muñoz-Santos C, Galván-Casas C, Roncero Riesco M, Revilla Nebreda D, Solá-Truyols A, et al. Cutaneous reactions after SARS-CoV-2 vaccination: a cross-sectional Spanish nationwide study of 405 cases. British Journal of Dermatology. 2021.

22. Juárez Guerrero A, Domínguez Estirado A, Crespo Quirós J, Rojas-Pérez-Ezquerra P. Delayed cutaneous reactions after the administration of mRNA vaccines against COVID-19. J Allergy Clin Immunol Pract. 2021.

23. Larson V, Seidenberg R, Caplan A, Brinster NK, Meehan SA, Kim RH. Clinical and histopathological spectrum of delayed adverse cutaneous reactions following COVID-19 vaccination. Journal of cutaneous pathology. 2021.

24. Lopatynsky-Reyes EZ, Acosta-Lazo H, Ulloa-Gutierrez R, Avila-Aguero ML, Chacon-Cruz E. BCG Scar Local Skin Inflammation as a Novel Reaction Following mRNA COVID-19 Vaccines in Two International Healthcare Workers. Cureus. 2021;13(4).

25. Peigottu MF, Ferreli C, Atzori MG, Atzori L. Skin Adverse Reactions to Novel Messenger RNA Coronavirus Vaccination: A Case Series. Diseases (Basel, Switzerland). 2021;9(3).

26. Sprute R, Schumacher S, Pauls M, Pauls W, Cornely OA. Delayed Cutaneous Hypersensitivity Reaction to Vaxzevria (ChAdOx1-S) Vaccine against SARSCoV-2. Drugs in R and D. 2021

27. Tihy M, Menzinger S, André R, Laffitte E, Toutous-Trellu L, Kaya G. Clinicopathological features of cutaneous reactions after mRNA-based COVID-19 vaccines. Journal of the European Academy of Dermatology and Venereology. 2021.

28. Lospinoso K, Nichols CS, Malachowski SJ, Mochel MC, Nutan F. A case of severe cutaneous adverse reaction following administration of the Janssen Ad26.COV2.S COVID-19 vaccine. JAAD case reports. 2021;13:134-7.

29. Eid E, Abdullah L, Kurban M, Abbas O. Herpes zoster emergence following mRNA COVID-19 vaccine. Journal of Medical Virology. 2021.

30. Chopra S, Kim Y, Flamm A. Cutaneous skin manifestation following messenger RNA Moderna SARS-CoV-2 vaccine with dermal hypersensitivity reaction histopathology. JAAD case reports. 2021;16:24-5.

31. Niebel D, Novak N, Wilhelmi J, Ziob J, Wilsmann-Theis D, Bieber T, et al. Cutaneous adverse reactions to covid-19 vaccines: Insights from an immunodermatological perspective. Vaccines. 2021;9(9).

32. Rice SM, Ferree SD, Mesinkovska NA, Kourosh AS. The art of prevention: COVID-19 vaccine preparedness for the dermatologist. International journal of women's dermatology. 2021.

33. Sun Q, Fathy R, McMahon DE, Freeman EE. COVID-19 Vaccines and the Skin: The landscape of cutaneous vaccine reactions worldwide. Dermatologic Clinics. 2021.

34. Fernandez-Nieto D, Hammerle J, Fernandez-Escribano M, Moreno-del Real C, Garcia-Abellas P, Carretero-Barrio I, et al. Skin manifestations of the BNT162b2 mRNA COVID-19 vaccine in healthcare workers.'COVID-arm': a clinical and histological characterization. Journal of the European Academy of Dermatology and Venereology. 2021;35(7):e425.

35. Baden LR, El Sahly HM, Essink B, Kotloff K, Frey S, Novak R, et al. Efficacy and safety of the mRNA-1273 SARS-CoV-2 vaccine. New England Journal of Medicine. 2021;384(5):403-16.

36. Polack FP, Thomas SJ, Kitchin N, Absalon J, Gurtman A, Lockhart S, et al. Safety and efficacy of the BNT162b2 mRNA Covid-19 vaccine. New England Journal of Medicine. 2020.

37. Klein SL, Flanagan KL. Sex differences in immune responses. Nature Reviews Immunology. 2016;16(10):626-38.

38. Fink AL, Klein SL. Sex and gender impact immune responses to vaccines among the elderly. Physiology. 2015.

39. Sako EY, Rubin A, Young LC. Localized bullous fixed drug eruption following yellow fever vaccine. Journal of the American Academy of Dermatology. 2014;70(5):e113-e4. 
40. Kelso JM. COVID-19: Allergic reactions to SARS-CoV-2 vaccines. Available from: https://wwwuptodatecom/contents/covid-19-allergic-reactions-to-sarscov-2-vaccines/print (Accessed on 14 October 2021).

\section{Figures}

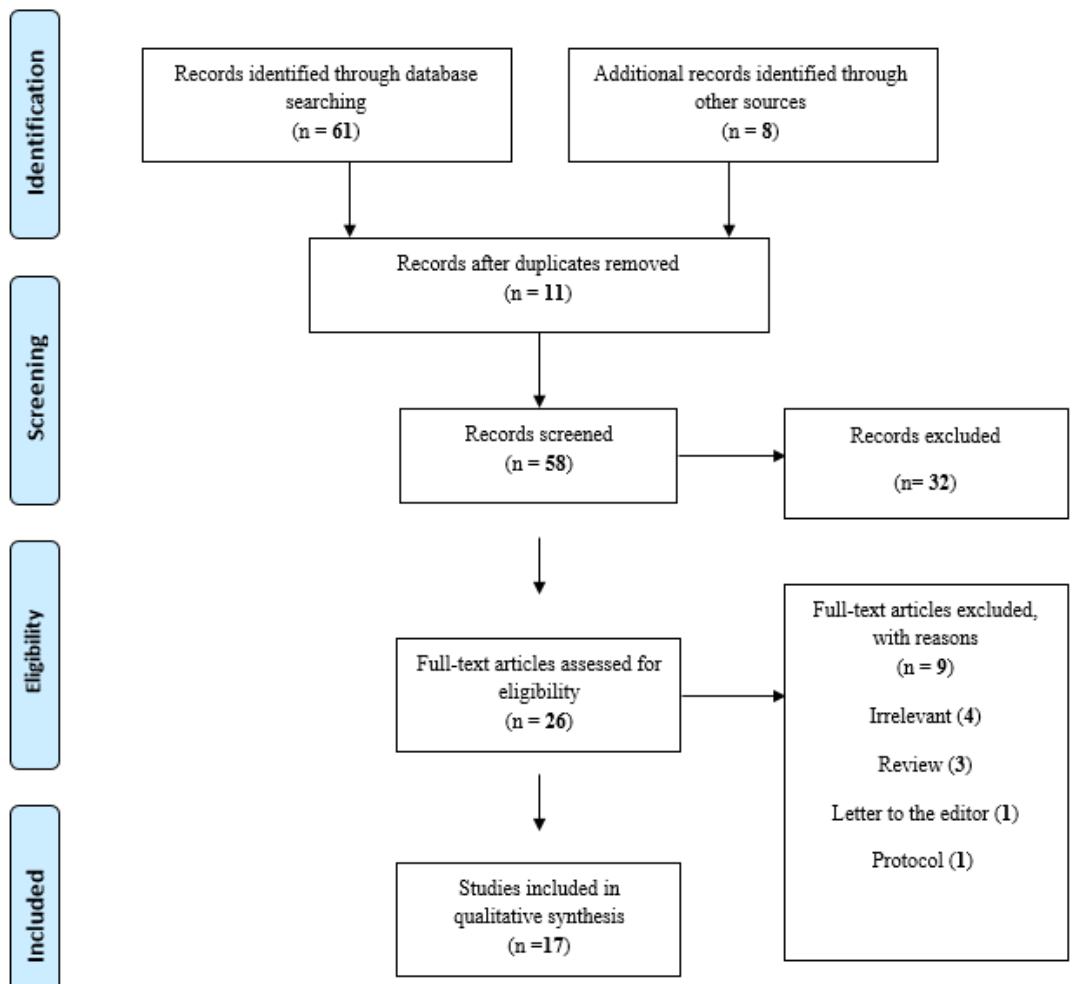

Figure 1

Search results from different databases 